\title{
A REVIEW ON PERFORMANCE INVESTIGATION OF ROUTING PROTOCOLS IN WIRELESS SENSOR NETWORKS
}

\section{SarojiniYarramsetti, ${ }^{2 \cdot}$ Anvar Shathik J, ${ }^{3 \cdot}$ Renisha P. S}

1. Associate Professor, Department of CSE, School of Engineering and Technology, Jain (To Be Deemed) University, Bangalore, India.

2. Associate Professor, Department of CSE, Srinivas University College of Engineering and Technology, Mangalore, India.

3. Assistant Professor, , Department of CSE, Srinivas University College of Engineering and Technology, Mangalore, India

\begin{abstract}
:
Wireless sensor networks are interconnected sensors used in large areas that can hear, process and communicate data over a large distance. Today sensor devices have found their use in defense work especially military services, medical services, disaster management, wildlife monitoring and precision farming in residential monitoring and logistics applications. As the sensor devices are smaller so they have limited energy resources that shorten their life. Due to this limitation, power management in these networks has become a major research area. This paper provides an overview of the performance of the five routing systems namely Ad hoc On-Demand Distance Vector (AODV), Temporally Ordered Routing Algorithm (TORA), Optimized Link State Routing Protocol (OLSR), Geographical Routing Protocol (GRP), Distance Routing Effect Algorithm for Mobility (DREAM) and their comparisons.
\end{abstract}

Keywords: AODV, DREAM, GRP, OLSR, Routing Protocols, TORA.

\section{INTRODUCTION:}

The Wireless Nerve Network consists of self-regulating, independent, low-energy sensors that work collaboratively in complex physical situations. They sense changes in visual cues such as movement, temperature and sound, translate small changes into data packets and transmit these packets of useful information through networks [1]. Considering the limitations of power in sensory nodes and the need for reduced transmission of large amounts of information to prevent energy loss, the availability of algorithms to be distributed and to process local information is of paramount importance [2]. A power source usually has a limited battery capacity. The development of a wireless sensory network was initially motivated by military programs such as battlefield surveillance. However, WSNs are now used in many areas of public applications that include the environment and accommodation 
monitoring. Due to the various limitations arising from their inexpensive nature, the limited size, weight and ad-hoc delivery method of each sensor faces the challenge of limited energy resources [3]. Various methods have been used to improve power consumption both at hardware level and software in Wireless Sensor Networks [4]. Different route schemes are designed to improve performance parameters. These router protocols are generally specific to their operation and do not work in a variety of applications. This paper selected three protocols for the route TORA, AODV and DREAM and attempted to analyze the operating parameters of these systems.

\section{ROUTING PROTOCOLS IN WSN:}

There are many router protocols built for wireless nerve networks that have the ability to manage resources especially in terms of energy efficiency, power distribution in a wide range of environments. In general, energy-saving protocols in WSNs can be divided into four categories and further subdivided into the many types given below in Figure 1.

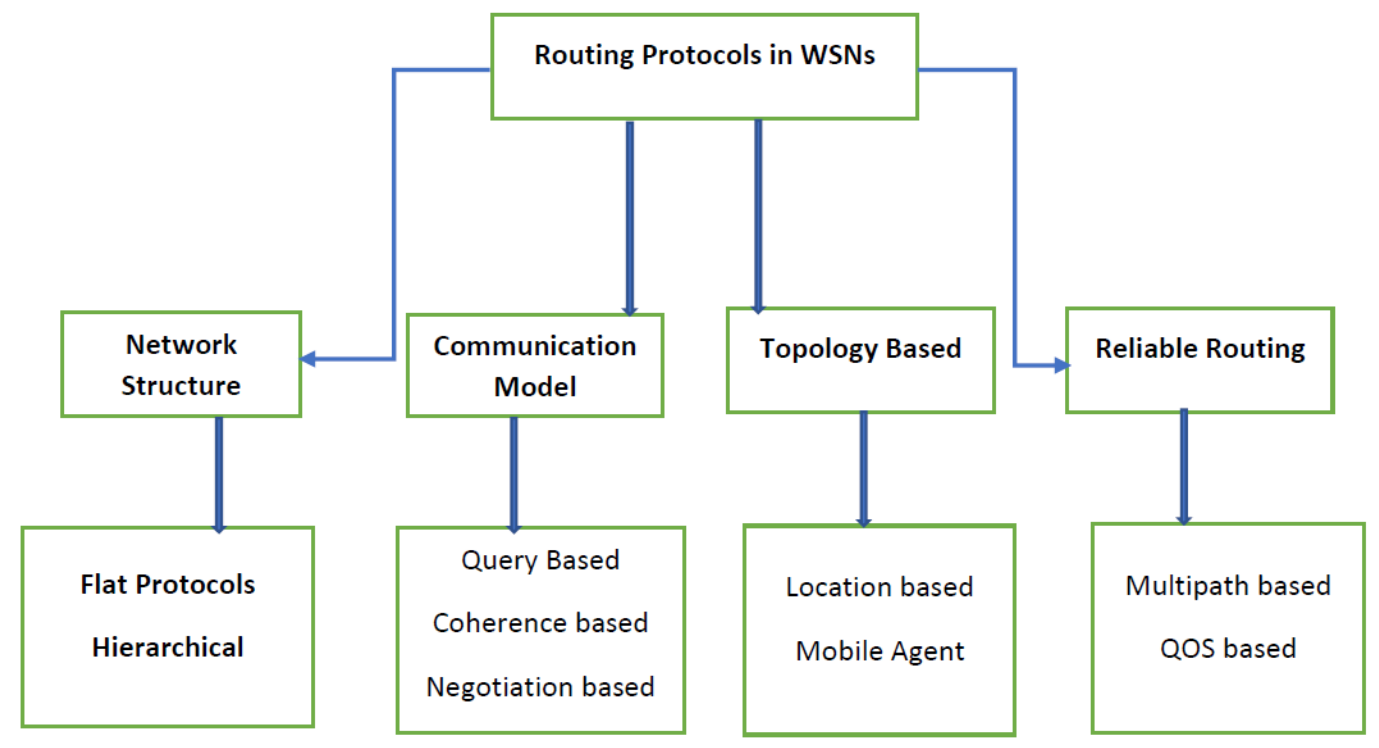

Figure 1 Routing Protocols in WSN

From the enormous number of routing protocols, we selected the following three routing protocols for the simulation purposes.

TORA: Temporally ordered routing algorithm (TORA) is a reactive, highly adaptive, efficient and scalable distributed routing algorithm based on the concept of link reversal. TORA is proposed for highly dynamic mobile, multi-hop wireless networks. It is a sourceinitiated on-demand routing protocol. It finds multiple routes from a source node to a destination node. The main feature of TORA is that the control messages are localized to a very small set of nodes near the occurrence of a topological change [5].

AODV: AODV Route Protocol is an active router protocol that establishes a route where a site needs to send data packets. AODV is capable of both unicast and multicast. The 
performance of the protocol is divided into two functions: route acquisition and route preservation [6]. AODV Route protocol is designed for mobile ad networks with a population of tens of thousands to thousands of mobile nodes. AODV can manage low, medium, and high transit rates compared to different data traffic levels [7]. Routes are established on demand and destination tracking numbers are used to find the latest route to the destination. Has Low Connection Setup Delay [8].

DREAM: The Distance Routing Effect Algorithm for Mobility (DREAM) is a location-based protocol framework with each node that stores a local database that stores location information about each node that is part of a network [9]. The DREAM protocol uses a completely different approach unlike the criteria mentioned above. DREAM features a GPS system that lets each location know its location. This makes the DREAM protocol very reliable. The advantage of exchanging location information is that it consumes much less bandwidth than exchanging full link status or distance vector information, making the legal process much faster [10].

OLSR: The Optimized Link State Routing ProtocolOLSR is a development of a pure link status algorithm [11], using the concept of Multi point Relays (MPR) to advance control traffic, which is intended to spread across the network. The MPR set is selected in such a way that it covers all nodes at a distance of two hops. Due to the nature of the operation, the OLSR operates from time to time with the exchange of messages such as Hello and Topology Control (TC) message via its MPR only. Parameters used by OLSR to control overheads of the protocol overhead Hello-interval parameter, TC interval parameter, MPR cover parameter and TC-redundancy parameter. Therefore, in contrast to the old link status algorithm, instead of all links, only small sets of links [12] are announced. In the pure connection mode the algorithm nodes are announced and filled the entire network. The OLSR protocol is the configuration of the MANETS pure link protocol. Reduce the size of the control packs instead of all the links and its neighbors who are its multi-point selectors that reduces the fullness of this traffic control by using only selected nodes called multipoint relays to transmit their messages over the network. Only many node points resume their broadcast messages. This process greatly reduces the number of relapses in the flood or distribution process.

GRP: Geographical Routing Protocol, a functional router system that uses the Global Positioning System (GPS) to locate a node location to collect network information at a source location with very little control over it. The source area can find routes and always transmit data even when the current route is closed. This approach is known as the hybrid route protocol due to the simultaneous use of the power of effective and efficient route agreements. A data packet called DQ is often used to transfer to each node of each node until it is reached. When it arrives at its destination, its destination announces its network information collection (NIG) package to its neighbors. The source area calculates the complete route according to the information collected and immediately begins to transfer data packets [13]. 


\section{SIMULATION SETUP AND RESULTS}

For simulation purposes OpnetModeller 17.5 Academic edition is used and the sensor networks used are wireless LAN workstations (mobile nodes) as they act same as that of any wireless sensor nodes. The entire work in this paper is divided into three modules and four scenarios; each scenario runs on the same set of sensor nodes while routing protocols are changed. Light traffic in module one and heavy traffic in module two has been incorporated. In module 1 we simulated AODV and TORA protocols and in Module 2 we simulated DREAM and TORA. In Module 3 under scenario 1 two more routing protocols namely OLSR and GRP have been added and simulation results have been recorded for light traffic. The scenario 2 of this module has recorded simulation results for different parameters for routing protocols namely AODV, TORA, OLSR and DREAM under heavy traffic. As shown in figure 2 and figure 3 , each scenario thereby is made of wireless sensor network comprising of wireless sensor nodes (wireless LAN workstations), A wireless LAN server, Application configuration object, Profile configuration object and Mobility configuration object. Then simulation results of selected algorithms in reference to the light traffic and heavy traffic are compared separately. The description of the modules and their corresponding scenarios are given below.

Module 1.

Scenario 1: AODV with light traffic

Scenario 2: TORA with light traffic

Module 2.

Scenario 3: DREAM with heavy traffic

Scenario 4: TORA with heavy traffic

Module 3.

Scenario 1: Comparison of AODV-TORA-OLSR-GRP.

Scenario 2: Comparison of AODV-TORA-OLSR-DREAM.

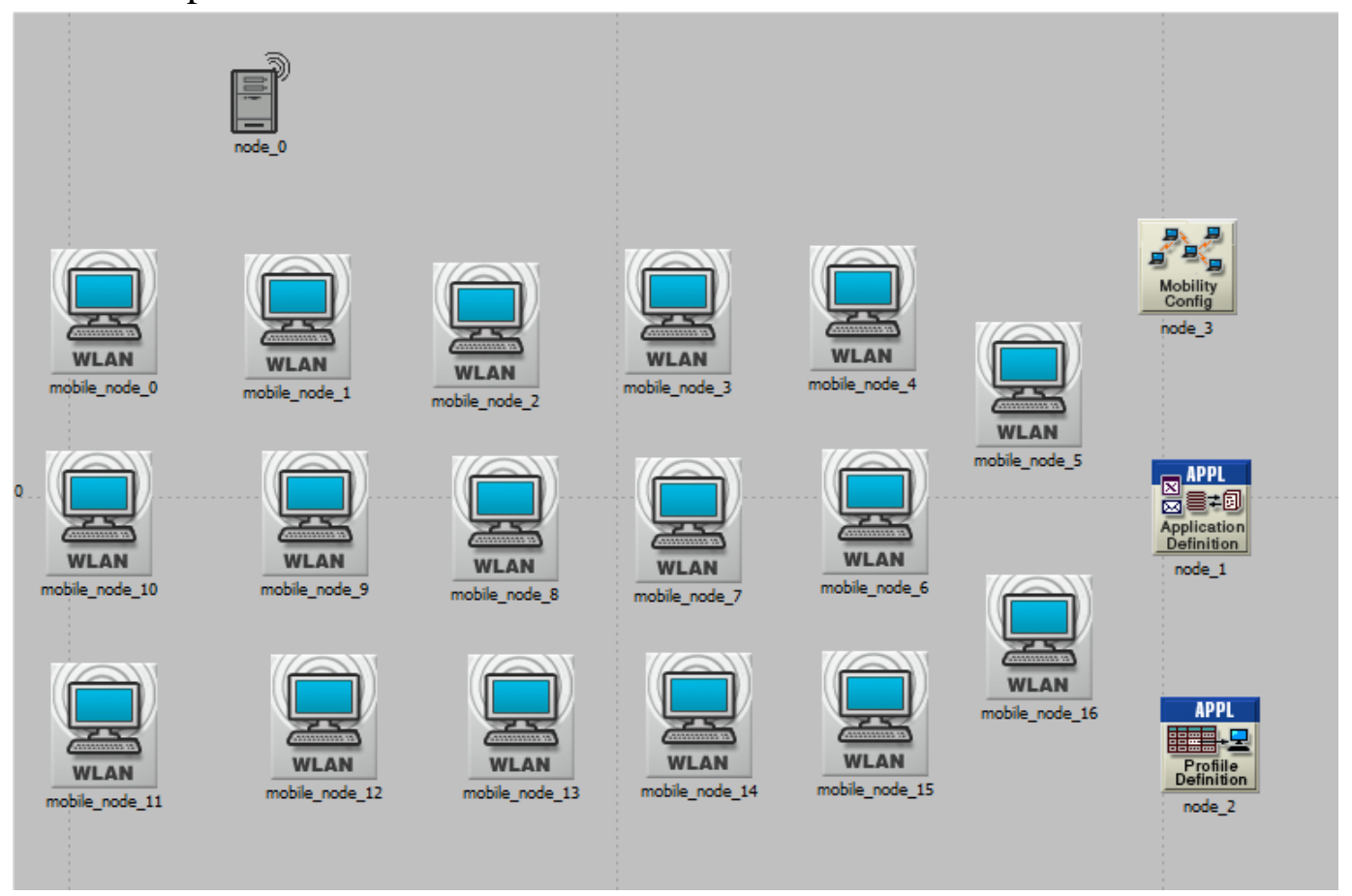

Figure 2 Light Traffic incorporation 
With the help of application and profile configuration objects we incorporated network traffic. A profile named PROF1 was created using profile configuration object which was configured to support only the file transfer protocols packets and with application configuration object, the application definition was set on FTP, so that the nodes could be configured for only FTP traffic. The sensor nodes will now be able to access the FTP traffic from the wireless LAN server.

The paths followed by the sensor nodes to receive and send the traffic/packets were defined using the mobility configuration object.

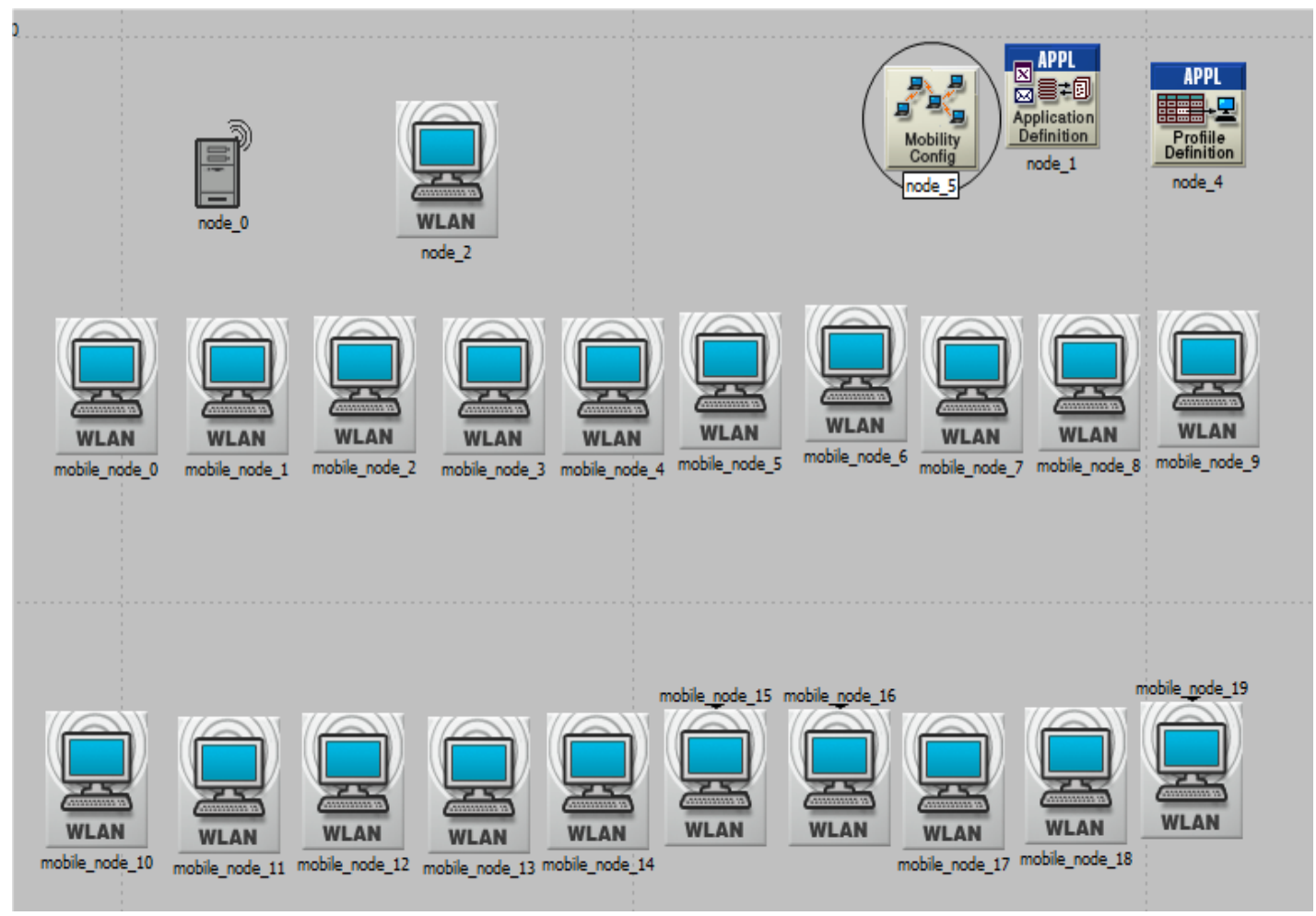

Figure 3 Heavy Traffic incorporation

Performance Metrics:

a. Throughput: Throughput can be defined as the data packets a node can process in a fixed interval of time. In terms of communication network, it is the rate at which data packets reached at the destination node successfully. It can be measured in bits per second.

b. Delay (End to End Delay): Delay is defined the time taken by a bit of data to go from transmission node to destination node in a network. Delay depends upon the location of nodes in a network and routing protocols.

c. Traffic Received (Data Packet Received):It can be defined as the data packets travelled across the network and reached at the destination node in a network.

d. Load:Load can be defined as the traffic across the network.

\section{Module 1}

In first scenario of module one, we configured the wireless sensor nodes for light traffic with the help of application and profile configuration object, the server and the nodes were 
configured to run only on the AODV routing protocol. And the second scenario was also configured using the wireless sensor nodes for light traffic with the help of application and profile configuration object, the wireless LAN server and the sensor nodes were configured to run only on the TORA routing protocol.

Module 2

In first scenario of module two, we configured the wireless sensor nodes for heavy traffic with the help of application and profile configuration object, the server and the nodes were configured to run only the TORA routing protocol. And the second scenario was also configured using the wireless sensor nodes for heavy traffic with the help of application and profile configuration object, the wireless LAN server and the sensor nodes were configured to run only the DREAM routing protocol. Then for all the four parameters throughput, delay, traffic received and load system was simulated.

Delay:From the study of the graph we find that among all the protocols used, OLSR is the most efficient in terms of delay, this protocol has lowest delay among all the other protocols used with almost $0 \%$ delay. TORA is having maximum delay of an average 0.0020 seconds.

Traffic Received: From the study of the graph, it is clearly visible that at the beginning of the simulation there is a sharp increase in the traffic received by all the networks, the network with TORA implementation receives maximum traffic. But there is a shift from maximum value to a more constant trend of traffic received in all the networks. TORA remains constant at $60000 \mathrm{bits} / \mathrm{sec}$ followed by OLSR with a value of $50000 \mathrm{bits} / \mathrm{sec}$. This shows the efficiency of TORA routing protocol in case of traffic received.

Load: The graph below presents a comparison Load (bits/sec). On an average load received by TORA is maximum than all the other routing protocols at an average value of 2000 bits/sec. the load value shows the efficiency of TORA to withstand and carry a heavy traffic by easy and efficient way. The next efficient routing protocol is AODV with a value close to TORA at almost $1800 \mathrm{bits} / \mathrm{sec}$. The fluctuations in all the protocols are considerably negligible. OLSR protocol has also a comparably efficient load capacity.

Table1 Values of parameters on light traffic under simulation

\begin{tabular}{|l|l|l|l|l|}
\hline Parameter studied & TORA & OLSR & AODV & GRP \\
\hline Throughput(bits/sec) & 170,000 & 50,000 & 60,000 & 59,000 \\
\hline Delay (seconds) & 0.0015 & 0.0001 & 0.0003 & 0.0004 \\
\hline Load (bits/sec) Average & 90,000 & 72,000 & 68,000 & 64,000 \\
\hline Data traffic Received (bits/sec) & 60,000 & 50,000 & 40,000 & 38,000 \\
& & & & \\
\hline
\end{tabular}


Module 3: Scenario2:

In this scenario, comparison of AODV, OLSR, TORA, OLSR and DREAM based on four performance factors shown below.

Throughput: The graph in figure 16 below shows the comparison of throughput for all the routing protocols. From the study of the graph obtained we can observe that the average throughput is maximum in case of DREAM routing protocol, followed by TORA and AODV. Moreover, the graph obtained shows that the throughput of DREAM is almost going linear and ends at a maximum value of $60000 \mathrm{bits} / \mathrm{sec}$ at the end of simulation. TORA protocol starts at a high value but is not able to keep a track of its progress and ends at a low value of almost $42000 \mathrm{bits} / \mathrm{sec}$. OLSR routing protocol maintains its value of 30000-40000 bits/sec throughout the simulation time of 1 hour.

Delay: From the study of the graph in figure 17, we could easily conclude that delay is less in case of AODV at a value of 0.0010 seconds, delay in case of DREAM is at a high value of 0.0045 seconds but the peculiar feature is that it starts at a low value of 0.0004 seconds and ends at the high delay value. OLSR remains constant at a value of 0.0015 seconds.

Traffic Received: The comparison of routing protocols for Data traffic received. From the graph, it can be observed that TORA has gained the highest value of traffic received but after almost 20 minutes of simulation it has stopped receiving further data, same is the case with AODV and OLSR, but only DREAM protocol has maintained its constant value of data reception throughout the simulation process. The DREAM protocol has maintained a constant value of $50000 \mathrm{bits} / \mathrm{sec}$ for a whole 1 hour of simulation time.

Load:The comparison of routing protocols on the selected parameter of Load (bits/sec). DREAM is seen as having the maximum load while AODV is having lowest as compared to other routing protocols.

Table 2 Values of parameters on heavy traffic under simulation

\begin{tabular}{|l|l|l|l|l|}
\hline & & & & \\
\cline { 2 - 5 } Parameter studied & TORA & OLSR & AODV & DREAM \\
\hline Throughput(bits/sec) & 45,000 & 40,000 & 40,000 & 60,000 \\
\hline Delay (seconds) & & & & \\
\hline Load (bits/sec) Average & 0.0038 & 0.0015 & 0.0010 & 0.0045 \\
\hline Data traffic Received (bits/sec) & 800,000 & 700,000 & 600,000 & $1,000,000$ \\
\hline
\end{tabular}




\section{DISCUSSIONS}

For module one it is seen that TORA with an average throughput of almost $2000 \mathrm{bits} / \mathrm{sec}$ is ahead of AODV protocol and fairs well in this parameter. In terms of delay TORA is having maximum delay of an average 0.0020 seconds, which is more than AODV protocol. In terms of traffic received TORA implementation receives maximum traffic. But there is a shift from maximum value to a more constant trend of traffic received in all the networks. TORA remains constant at $60000 \mathrm{bits} / \mathrm{sec}$ followed by AODV with a value of $50000 \mathrm{bits} / \mathrm{sec}$. This shows the enhanced efficiency of TORA routing protocol in case of traffic received and in terms of delay on an average load received by TORA is maximum than all the other routing protocols at an average value of $2000 \mathrm{bits} / \mathrm{sec}$. The load value shows the efficiency of TORA to withstand and carry a heavy traffic by easy and efficient way. The next efficient routing protocol is AODV with a value close to TORA at almost $1800 \mathrm{bits} / \mathrm{sec}$. The fluctuations in all the protocols are considerably negligible.

For second module, the average throughput is maximum in case of DREAM routing protocol, followed by TORA. Moreover, the graph obtained shows that the throughput of DREAM is almost going linear and ends at a maximum value of $60000 \mathrm{bits} / \mathrm{sec}$ at the end of simulation. TORA protocol starts at a high value but is not able to keep a track of its progress and ends at a low value of almost $42000 \mathrm{bits} / \mathrm{sec}$. In terms of delay we could easily conclude that delay is less in case of TORA at a value of 0.0010 seconds, delay in case of DREAM is at a high value of 0.0045 seconds but the peculiar feature is that it starts at a low value of 0.0004 seconds and ends at the high delay value. In terms of traffic received TORA has gained the highest value of traffic received but after almost 20 minutes of simulation it has stopped receiving further data, but only DREAM protocol has maintained its constant value of data reception throughout the simulation process. The DREAM protocol has maintained a constant value of $50000 \mathrm{bits} / \mathrm{sec}$ for a whole 1 hour of simulation time and in terms of load it is clear that DREAM routing protocol has the Maximum load value of $60000 \mathrm{bits} / \mathrm{sec}$ followed by TORA with $52000 \mathrm{bits} / \mathrm{sec}$. DREAM protocol has reached to a maximum value of 100000 bits/sec among all the other protocols.

For module three, it can be again proved that DREAM is the best routing protocol among all others discussed and simulated in this paper based on various performance metrics.

\section{CONCLUSION:}

This paper describes the comparison of different route protocols in the four parameters of small traffic and large traffic. In the case of low traffic traffic, TORA has proven to be an effective route protocol while in heavy traffic integration DREAM route protocol is proven to be the most efficient route protocol among all. Overall the graphs and results discussed in both low traffic and high traffic, DREAM are considered the applicable routes by TORA and AODV. 


\section{REFERENCES}

[1].Jamatia, A et al, "Performance Analysis of Hierarchical and Flat Network Routing Protocols in Wireless Sensor Network Using NS-2",International Journal of Modeling and Optimization, Vol. 5, No. 1, February 2015.

[2].Ilyas M. and Mahgoub I. "Handbook of Sensor Networks: Compact Wireless and Wired Sensing Systems", Proceedings of the CRC Press, CRC PRESS LLC, Boca Raton 2005.

[3].Rezaei Z., Mobininejad S."Energy Saving in Wireless Sensor Networks," International Journal of Computer Science \& Engineering Survey, Volume 3, No.1, February 2012.

[4].Mir. W, Ahmad.J, "Energy Management in Wireless Sensor Networks-A Review," International Journal of Advanced Research in Computer Science, Volume 8, No. 4, May June 2017.

[5].Khiavi. M.V, Jamali.S, Gudakahriz. S.J, "Performance Comparison of AODV, DSDV, DSR and TORA Routing Protocols in MANETs", International Research Journal of Applied and Basic Sciences. Volume 3 (7), 1429-1436, 2012.

[6].C. Perkins," Ad hoc On-Demand Distance Vector (AODV) Routing", Nokia Research Center E. Belding Royer University of California, Santa Barbara S. Das University of Cincinnati, July 2003.

[7].Kumar Sachin, Saket.R.K, "Performance Metric of AODV and DSDV Routing Protocols in MANETs Using NS-2 ",International Journal of Recent Research and Applied Studies, Volume 7 (3), June 2011.

[8].Mauve.M, Widmer.J, "A Survey on Position-Based Routing in Mobile Ad Hoc Networks", IEEE Network, Nov/Dec 2001.

[9]. Abolhasan, M., Wysocki, T. \&Dutkiewicz, E. "A review of routing protocols for mobile ad hoc networks". Ad Hoc Networks, 2(1), 1-22, 2004.

[10]. S.Mohapatra, P.Kanungo "Performance analysis of AODV, DSR, OLSR and DSDV Routing Protocols using NS2 Simulator", International Conference on Communication Technology and System Design, Volume 30, Pages 69-76, 2012.

[11]. Clausen, Thomas, and Philippe Jacquet. Optimized link state routing protocol (OLSR). No. RFC 3626. 2003.

[12]. Mittal, Puneet, Paramjeet Singh, and Shaveta Rani. "Performance analysis of AODV, OLSR, GRP and DSR routing protocols with database load in MANET." International Journal of Research in Engineering and Technology 2.9 (2013): 412-20.

[13]. Yassine, Maleh, and AbdellahEzzati. "Performance analysis of routing protocols for wireless sensor networks." Information Science and Technology (CIST), 2014 Third IEEE International Colloquium in. IEEE, 2014. 